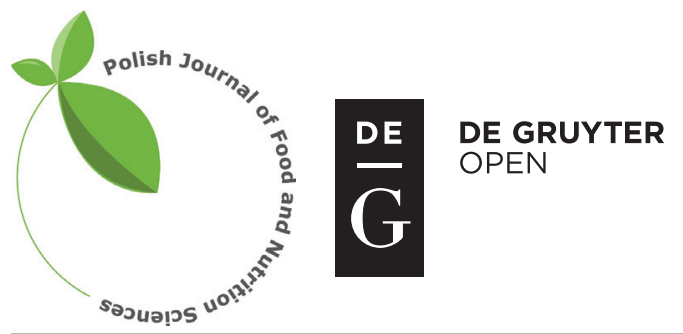

Pol. J. Food Nutr. Sci., 2018, Vol. 68, No. 2, pp. 149-162

DOI: $10.1515 /$ pjfns-2017-0023 http://journal.pan.olsztyn.pl

Original research article

Section: Food Quality and Functionality

\title{
Effect of Quercetin on Bone Mineral Status and Markers of Bone Turnover in Retinoic Acid-Induced Osteoporosis
}

\author{
Nada Oršolićl ${ }^{*}$, Željko Jeleč ${ }^{2}$, Johann Nemrava ${ }^{3}$, Vedran Balta ${ }^{1}$, Gordana Gregorovićt ${ }^{4}$, Dražen Jelečs \\ 'Department of Animal Physiology, Faculty of Science, University of Zagreb, Rooseveltov trg 6, 10000 Zagreb, Croatia \\ ${ }^{2}$ Department of Orthopaedic Surgery, Specialty Hospital St. Catherine, Zabok, Croatia \\ ${ }^{3}$ Department of Surgery and Emergency medicine, General Hospital Zabok, Croatia \\ ${ }^{4}$ Division of Biology, Faculty of Science, University of Zagreb, Rooseveltov trg 6, HR-10000 Zagreb, Croatia \\ ${ }^{5}$ Roche d.o.o., Zagreb, Croatia
}

Key words: secondary osteoporosis, bone remodeling markers, bone mineral density, quercetin, histology, rat

Retinoic acid-induced osteoporosis (RBM) is one of the most common causes of secondary osteoporosis. This study tested the anti-osteoporetic effect of quercetin in RBM-induced bone loss model (RBM). After 14-day supplementation of 13cRA to induce RBM, rats were administered with quercetin $(100 \mathrm{mg} / \mathrm{kg})$ or alendronate $(40 \mathrm{mg} / \mathrm{kg})$. We analysed changes in body and uterine weight of animals, femoral geometric characteristics, calcium and phosphorus content, bone weight index, bone hystology, bone mineral density (BMD), markers of bone turnover, lipid peroxidation, glutathione levels and SOD, CAT activity of liver, kidney spleen, and ovary as well as biochemical and haematological variables.

In comparison to the control RBM rats, the treatment with quercetin increased bone weight index, BMD, osteocalcin level, femoral geometric characteristics, calcium and phosphorus content in the 13cRA-induced bone loss model. Histological results showed its protective action through promotion of bone formation. According to the results, quercetin could be an effective substitution for alendronate in 13cRA-induced osteoporosis. Good therapeutic potential of quercetin on rat skeletal system is based partly on its antioxidant capacity and estrogenic activity.

\section{INTRODUCTION}

Osteoporosis is a metabolic disease characterized by loss of bone density and deterioration of bone microarchitecture, resulting in increased risk for bone fractures. Ageing, estrogen deficiency and oxidative stress are the contributing factors resulting into osteoporotic bone. In addition, certain medications increase the rate of bone loss [Pitts \& Kearns, 2011].

It is estimated that osteoporosis affects at least 200 million women worldwide [Johnell \& Kanis, 2006]. A recent World Health Organization report shows that osteoporosis is a global health problem as well as cardiovascular disease, cancer, and diabetes [Walker-Bone, 2012].

Treatment for osteoporosis is divided into primary and pharmacological by antiresorptive and osteoanabolic drugs. The primary treatment means to change bad habits (drinking, smoking), elimination of risk factors, adequate intake of vitamin $\mathrm{D}$ and calcium, healthy diet and regular physical activity. Recently, more attention is paid to diet rich in flavonoids, known as the phytoestrogens, which can stimulate osteoblastic bone formation and inhibit bone resorption [Liu

\footnotetext{
* Corresponding Author: Tel: +3851 4877 747; Fax: +385 14826 260; E-mail: norsolic@yahoo.com; nada.orsolic@biol.pmf.hr (Prof. dr. sc. N. Oršolić)
}

et al., 2015; Oršolić et al., 2014; Yang et al., 2016; Zhao et al., 2016].

Length and complexity of the ovariectomized model has led to the development of new osteoporotic models such as glucocorticoids or retinoic acid models [Bitto et al., 2009; Fahmy \& Soliman, 2009; Hozayen et al., 2016; Liu et al., 2015; Oršolić et al., 2014; Peng et al., 2005; Yang et al., 2016; Zhao et al., 2016]. So, application of high doses of vitamin A (retinoic acid) in a period of $1-3$ weeks causes the decrease of bone mass and histomorphometrical structural changes that correspond to osteoporosis [Fahmy \& Soliman, 2009; Nowak et al., 2016]. Proposed mechanisms for the occurrence of osteoporotic-like changes induced by retinoic acid are a decrease in estrogen level, increase in osteoclasts numbers and activities, enhanced bone resorption and depression of endochondral ossification that resulted from epiphyseal plate cartilage lesion as well as oxidative stress [Fahmy \& Soliman, 2009; Henning et al., 2015; Hozayen et al., 2016].

Quercetin is a naturally occurring flavonoid and potentially has beneficial effects on disease prevention including osteoporosis. It has been reported that quercetin inhibits bone loss in rat model of diabetic osteopenia and exerts protective effects against $\beta$-cell damage in diabetes [Coskun et al., 2005; Oršolić et al., 2011]. Quercetin is a powerful natural antioxidant and can protect many types of tissue or organs 
against oxidative damage, including liver, kidney and pancreatic gland [Knežević et al., 2011; Oršolić et al., 2011, 2016; Oršolić \& Car, 2014]. Furthermore, quercetin has beneficial effects on bone cells and tissues and it is capable of inhibiting bone loss in ovariectomized mice [Tsuji et al., 2009] and retinoic acid-induced bone loss [Oršolić et al., 2014].

However, little is known about the mechanisms of tissue-selective effects of quercetin, alendronate or retinoic acid on ovary. The aim of this study is to provide a better insight into the role of quercetin as an antioxidant and estrogen-like component, more specifically to demonstrate its efficacy in inhibiting drug-induced reactive radicals, consequent inflammation, estrogen deficiency and bone loss.

In this way, clinical practice will be given another option of osteoporosis treatment, which would probably be more accessible to a price and with far fewer side effects. In addition, quercetin could be a good substitute for alendronate, which induces a strong inflammatory effect and increases the risk of developing cancer in post-menopausal women [Chiang et al., 2012] and of atypical femoral fractures as defined by the American Society for Bone and Mineral Research (ASBMR) after long-term bisphosphonate therapy.

\section{MATERIAL AND METHODS}

\section{Animals}

Present study was approved by the ethical committee (Faculty of Science, University of Zagreb, Croatia). Three month-old female rats from highly fertile Y59 strain [http://www. informatics.jax.org/external/festing/rat/docs/Y59.shtml], weighing 200 to $250 \mathrm{~g}$, obtained from the Department of Animal Physiology, Faculty of Science, University of Zagreb, were used in this study. The animals were kept in individual cages during the experiment and at 12 hours of light per day. They were fed a standard laboratory diet (4 RF 21, Mucedola, Settimo Milanese, Italy) and tap water ad libitum. Maintenance and care of all experimental animals were carried out according to the guidelines in force in Republic of Croatia (Law on the Welfare of Animals, NN135/06 and NN37/13) and in accordance with EU Directive 2010/63/EU for animal experiments (reference: OJEU 2010) and carried out in compliance with the Guide for the Care and Use of Laboratory Animals, DHHS Publ. \# (NIH) 86-123.

\section{Reagents}

Isotretinoin (13cRA; 13 cis-Retinoic acid) (Roaccutane $\AA$, Hoffmann-La Roche Ltd, Basel, Switzerland) was used for induction of osteoporosis in rats at a dose of $80 \mathrm{mg} / \mathrm{kg}$. Alendronate (Alendor ${ }^{\circledR} 70$, Pliva, Croatia) was used as a positive control by intragastric application (ig) during 14 consecutive days at a dose of $40 \mathrm{mg} / \mathrm{kg}$ [Oršolić et al., 2014; Wei et al., 2007].

\section{Quercetin}

Quercetin (Quercetin dihydrate 98\%, Aldrich Ch. Co. Inc. Milwauke WI, USA), was used in the study. Before use, quercetin was dissolved in ethanol and further dilutions were made in water. The final concentration of ethanol was less than or equal to $0.5 \%(\mathrm{v} / \mathrm{v})$. Ethanol $(0.5 \%)$ was used in the control group. Quercetin was given to rats by intragastric application (ig) during 14 consecutive days at a dose of $100 \mathrm{mg} / \mathrm{kg}$ body weight [Oršolić et al., 2014].

\section{Experimental design - retinoic acid-induced rat osteoporosis and treatment}

A total of 60 Y59 female rats were used in the study. Three groups of rats (RBM, RBM+Quercetin, RBM+Alendronate) were administered $i g$ with retinoic acid suspension $(80 \mathrm{mg} / \mathrm{kg}$ BW) once daily for 1-14 experimental days (Figure 1). Groups

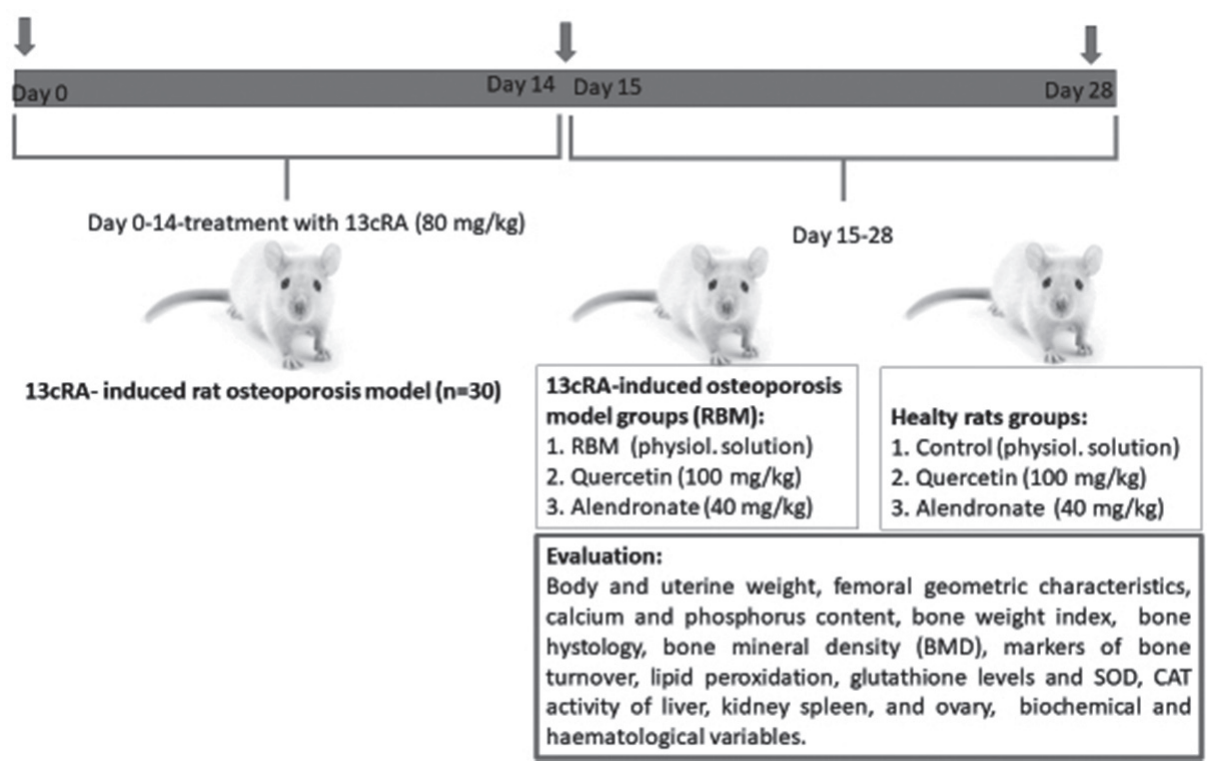

FIGURE 1. Experimental design. Three groups of rats (RBM, RBM+Quercetin, RBM+Alendronate) were administered $i g$ with retinoic acid suspension $(80 \mathrm{mg} / \mathrm{kg} \mathrm{BW})$ once daily for 1-14 experimental days. Groups Control, Quercetin and Alendronate were treated $i g$ with physiological solution during 1-14 days. Groups Quercetin and RBM+Quercetin were administered ig with quercetin (100 mg/kg BW) once daily during 15-28 days of the study. Groups Alendronate and RBM+Alendronate were administered ig alendronate (40 mg/kg BW) once daily during 15-28 days of the study. Number of rats per group: 7-10. Abbreviations: 13cRA - 13 cis-Retinoic acid; BW - body weight; RBM - 13cRA-induced bone loss model. 
Control, Quercetin and Alendronate were treated ig with a physiological solution with $0.5 \%$ ethanol during $1-14$ days. Groups Quercetin and RBM+Quercetin were administered ig with quercetin (100 mg/kg BW) once daily during 15-28 days of the study [Oršolić et al., 2014]. Groups Alendronate and $\mathrm{RBM}+$ Alendronate were administered ig alendronate (40 mg/kg BW) once daily during 15-28 days of the study [Oršolić et al., 2014; Wei et al., 2007]. Healthy animals prior to the experiment were fed with standard rodent food and occasionally caught and moved to another cage to have the same stress and conditions as the animals in the experiment. For the last 14 days, the control group of healthy animals received ig physiological solution while the remaining 2 groups received ig quercetin or alendronate.

All rats were fed and caged under consistent conditions during the study. The dosing was adjusted according to the status of the rat weight on a daily basis. At 4 weeks after treatments, the rats were anesthetized using a mixture of ketamine (Narketan ${ }^{\circledR} 10$, Vetoquinol AG, Belp Bern, Switzerland) at a dose of $80 \mathrm{mg} / \mathrm{kg}$ with xylazine (Xylapana ${ }^{\circledR}$ Vetoquinol Biowet Sp., Gorzow, R. Poland) at a dose of $10 \mathrm{mg} / \mathrm{kg}$ and exsanguinations was performed from axillary blood vessels for haematological and biochemical analyses. The blood was immediately placed on ice prior to isolation of the serum by centrifugation. The right and left femur were then harvested, carefully cleaned of soft tissue, weighed, and measured lengthwise using callipers. The right femurs were weighed for the analysis of bone weight index ( $\mathrm{g} / 100 \mathrm{~g}$ body weight) and for calcium (Ca) and phosphorus (P) analysis. The left femur was used for histological analysis and bone mineral density (BMD). The uterus of each rat was dissected and weighed. Liver, kidney, spleen and ovary tissues were used to determine oxidative and anti-oxidative status by measuring lipid peroxidation, glutathione levels, and SOD and CAT activity.

\section{Monitoring changes in weight of animals}

The animals are weighed on digital scales: i) at the beginning of the experiment; ii) during administration of retinoic acid and causing osteoporosis, iii) during the treatment of osteoporotic animals with quercetin or alendronate, and iv) at the end of the experiment. From these data, the mean change in body weight was calculated. The maximum percentage of animal weight loss, was calculated for individual animals as:

Percentage change in weight $=$ $=$ Final weight - Initial weight $\times 100 /$ Final weight

\section{Uterine weight}

The relative uterine weight was expressed in $\mathrm{g} / 100 \mathrm{~g}$ and was calculated as:

Relative uterine weight $(\mathrm{g} / 100 \mathrm{~g})=$ $=$ Total uterine weight $\times 100 /$ Final body weight

\section{Bone harvesting and measurements}

Immediately after sacrificing, the right hind leg of the rat was dissected. After removal of the flesh from the femur, they were then soaked in saline to prevent dehydration for subsequent measurement of bone wet weight, length, and bone diameters (physical dimensions). The femur wet weight was determined, to the nearest $0.01 \mathrm{mg}$, on an electrical balance (AA-160, Denver Instrument Company, U.S.A.) immediately after the bone harvesting. Means of the right and left femurs were used for the analysis of bone weight index (g/100 g body weight). The femur length was then measured, to the nearest $0.01 \mathrm{~mm}$, with a digital sliding calliper (A.S.M., Germany), from the top of the femur head to the distal point of the femur. In addition to femur length, the proximal epiphyseal diameters of the femur were measured in medio-lateral and anterior-posterior directions by digital calliper. The bones were then used for calcium $(\mathrm{Ca})$ and phosphorus $(\mathrm{P})$ analysis.

\section{Calcium (Ca) and phosphorus (P) analysis}

For the calcium $(\mathrm{Ca})$ and phosphorus $(\mathrm{P})$ analysis, dissected right femurs were rinsed with deionized water, dried at $105^{\circ} \mathrm{C}$ and then dry-ashed overnight in a muffle furnace at $450^{\circ} \mathrm{C}$ (Gallenkamp, England). The ash residues were dissolved in concentrated nitric acid $\left(\mathrm{HNO}_{3}, 65 \%\right.$, p.a. purity), heated and filled up after cooling to $10 \mathrm{~mL}$ with deionized water as described in Oršolić et al. [2014].

Determination of Ca was performed by aspirating diluted sample solution into the air-acetylene flame of SpectrAA-300 (Varian, Australia) and using lanthanum as a matrix modifier. At least two replicate determinations were made for each sample. Results are expressed as $\mathrm{mg} \mathrm{Ca/g}$ of wet tissue weight.

Phosphorus was determined by modified spectrophotometric method [Oršolić et al., 2014]. The analyte was reduced and deproteinized by trichloroacetic acid containing ferrous ion and thiourea and phosphate content was estimated by adding a molybdate solution. Formed phosphomolybdate is immediately reduced in situ by the ferrous ion to produce a blue color that is stable within $2 \mathrm{~h}$. The absorbance of the unknown samples and standard was measured spectrophotometrically at $660 \mathrm{~nm}$ (Cary 50; Varian, Australia). The results are expressed as $\mathrm{mg} \mathrm{P/g}$ of wet tissue weight.

Accuracy of the methods was evaluated using Animal bone reference material (H5, International Atomic Energy Agency, Austria). The results of our analysis were within $\pm 10 \%$ of the reference values, i.e. $214.02 \pm 1.81 \mu \mathrm{g} / \mathrm{g}$ for Ca, reference value $212 \mu \mathrm{g} / \mathrm{g}$ wet weight and $102.29 \pm 0.31 \mu \mathrm{g} / \mathrm{g}$ for $\mathrm{P}$, reference value $102 \mu \mathrm{g} / \mathrm{g}$ wet weight.

\section{Measurement of bone mineral density (BMD)}

The right femurs after quarrying were stored in test tubes in a freezer to determine bone mineral density (BMD).

The area of proximal and distal parts of the left neck of femur (thigh bone metaphysis) were subjected to measurement to obtain the values of bone area $\left(\mathrm{cm}^{2}\right.$-area) and bone mineral content (BMC, g). Data on bone mineral density (BMD) were calculated from the relationship of these parameters $\left(\mathrm{g} / \mathrm{cm}^{2}\right)$.

The method used to measure these parameters (area, BMC, BMD) was dual energy X-ray densitometric absorptiometry Hologic on apparatus QDR ${ }^{\circledR} 4000$ (Hologic Inc., Zaventem, Belgium) with appropriate software for small animals (1.0. high resolution). All BMD measurements were performed by the same technician. During the analysis of these parameters, the manufacturer's instructions were followed in order to assess long-term reproducibility of the measured 
values. The coefficient of variation (QC) for BMD and femoral bone amounted to $1.15 \%$ or $1.1 \%(0.61 \%)$.

\section{Serum markers of bone turnover}

The serum levels of bone turnover markers, including osteocalcin (OC), and $\beta$-CrossLaps ( $\beta$-CTx), were determined. Serum osteocalcin, a marker of bone formation, was measured using an N-MID Osteocalcin kit (Cobas-Roche, Switzerland), while serum $\beta$-CTx, a marker of bone resorption, was measured by a $\beta$-CrossLaps kit (Cobas-Roche, Switzerland). Serum osteocalcin and $\beta$-CTx were measured by electrochemiluminescence "ECLIA" method by Elecsys 2010 immunoassay analyzers (Hitachi High-Technologies Corporation, Tokyo, Japan) according to the manufacturers' instructions.

\section{Histological analysis}

After the rats were sacrificed, the left femurs were removed, cleared from attached muscle tissue and fixed in 10\% neutral buffered formalin. The bone specimens were then washed thoroughly in tap water for $30 \mathrm{~min}$ and then placed in decalcifying fluid RDO (Apex Engineering Products; Plainfield, IL) for $24 \mathrm{~h}$. After decalcification, each of the bones was cut at the mid shaft of its diaphysis and then rinsed thoroughly in slowly running tap water, dehydrated in a graded alcohol series $(70 \%, 80 \%, 96 \%$, and $100 \%)$ and after chloroform treatment embedded in paraplast. Deparaplasted 6-7 $\mu \mathrm{m}$ thick cross sections of the femur distal diaphysis were stained with hematoxylin and eosin (HE) following a standard protocol. Stained sections taken from comparable areas were examined under a light microscope (Nikon Eclipse E600) at 40, 100 and 200x magnification. Photomicrographs were taken by digital camera (Nikon DMX1200) and Imaging Software Lucia G 4.80 (Laboratory Imaging Ltd., Prague, Czechoslovakia).

\section{Analysis of hematological and biochemical parameters}

Blood was withdrawn from the axillary plexus of blood vessels of experimental animals and placed in heparinized glass vacutainer with the addition of $\mathrm{K}_{3}$ EDTA (Becton Dickinson, Plymouth, United Kingdom) and kept at the temperature from $4^{\circ} \mathrm{C}$ for $2-4 \mathrm{~h}$ to determine the hematological parameters.

For the evaluation of hematological parameters, $1 \mathrm{~mL}$ of blood was taken into a test tube with a vacuum with the addition of anticoagulants $\mathrm{K}_{3}$ EDTA. For the assessment of biochemical parameters, blood samples were collected into tubes without anticoagulant, the clotting of blood serum was used for the analysis of biochemical parameters. The coagulate of blood was centrifuged at $3000 \mathrm{rpm}$ for $10 \mathrm{~min}$ and then serum was collected into $1.5 \mathrm{~mL}$ polypropylene tubes (Eppendorf AG, Hamburg, Germany) and stored at $-20^{\circ} \mathrm{C}$ until determination of biochemical parameters.

From the hematological parameters there were determined: number of erythrocytes (E), the average cellular volume of erythrocytes (MCV), hemoglobin (Hgb), hematocrit (Hct), mean cell hemoglobin ( $\mathrm{MCH})$, mean cell hemoglobin concentration (MCHC), total leukocyte count (L), and the total number of platelets (Plt). From the standard biochemical parameters there were determined aspartate aminotransferase (AST), alanine aminotransferase (ALT), alkaline phosphatase (ALP), urea, creatinine, blood glucose levels (glucose), lactate dehydrogenase (LDH), and total protein.

Blood samples were analyzed with standard laboratory methods. Hematologic parameters, were analyzed using a Cell-Dyn (® 3700 blood cell counter (Abbott, USA), and a Becmann Coulter AU 680 biochemical apparatus (USA).

\section{Tissue preparations for quantitative analysis of antioxidant enzyme activities}

Portions of liver, kidney, spleen and ovary samples of $100 \mathrm{mg}$ were homogenized in $1 \mathrm{~mL}$ of $50 \mathrm{mmol} / \mathrm{L}$ phosphate buffer $(\mathrm{pH}$ 7.0) by a SONOPLUS Bandelin HD2070 ultrasonic homogenizer (Bandelin, Germany) using an MS73 probe (Bandelin, Germany) with a power of $10 \%$. Homogenates were centrifuged by a Micro 200R centrifuge (Hettich, Germany) for $15 \mathrm{~min}$ at a speed of 10,000 rpm at $+4^{\circ} \mathrm{C}$. The supernatant was used for measurements of glutathione and lipid peroxidation levels, superoxide dismutase (SOD) and catalase (CAT) activity. All methods were described before [Oršolić et al., 2016]. All parameters were normalized in relation to exact protein content.

\section{Measurement of lipid peroxidation}

Lipid peroxidation was determined by measuring the concentration of malondialdehyde (MDA) that reacts with thiobarbituric acid and produces chromogen that is measured spectrophotometrically at $532 \mathrm{~nm}$ and $600 \mathrm{~nm}$ with Libro S22 spectrophotometer (Biochrom, UK) as described in Oršolić et al. [2016].

\section{Total glutathione assay}

The glutathione level was determined by 5-5'-dithiobis [2-nitrobenzoic acid] (DTNB, Ellman's Reagent) and measured on the spectrophotometer at $412 \mathrm{~nm}$ as described before [Oršolić et al., 2016].

\section{Measurement of total superoxide dismutase (SOD) activity}

SOD activity was calculated from the percentage of inhibition of the reaction of xanthine oxidation by xanthine oxidase (optimized reaction ratio $\Delta \mathrm{A} / \min \approx 0.025$ ), which generates a superoxide anion as a substrate for SOD present in the samples. The percentage of inhibition was calculated using a calibration curve of different dilutions of SOD enzyme values as detailed in the paper by Oršolić et al. [2016].

\section{Measurement of catalase (CAT) activity}

In this method, catalase activity was determined by decrease in $\mathrm{H}_{2} \mathrm{O}_{2}$ absorbance at $240 \mathrm{~nm}$. The reaction mixture contained $10 \mathrm{mmol}_{2} \mathrm{O}_{2}$, in phosphate buffer $(\mathrm{pH}$ 7.0), and requisite volume of the sample in a total volume of $1 \mathrm{~mL}$. Catalase activity was measured by the extinction coefficient of $\mathrm{H}_{2} \mathrm{O}_{2}\left(\varepsilon=39.4 \mathrm{mM}^{-1} \mathrm{~cm}^{-1}\right)$; the specific activity was calculated and was expressed as $\mathrm{U} / \mathrm{mg}$ protein. 
TABLE 1. Effects of quercetin or alendronate on the changes in body weight and bone weight index in non-osteoporotic rats and rats with retinoic acid-induced bone loss.

\begin{tabular}{l|c|c|c}
\hline \multirow{2}{*}{ Treatment $^{\mathrm{a}}$} & \multicolumn{2}{|c|}{ Changes in body weight $(\%)$} & \multirow{2}{*}{$\begin{array}{c}\text { Bone weight } \\
\text { index }\end{array}$} \\
\cline { 2 - 3 } & $\begin{array}{c}\text { 1-14 days } \\
\text { (Mean } \pm \text { SEM) }\end{array}$ & $\begin{array}{c}15-28 \text { days } \\
\text { (Mean } \pm \text { SEM) }\end{array}$ & (Mean \pm SEM) \\
\hline Control & $2.80 \pm 0.400$ & $5.94 \pm 0.570$ & $0.395 \pm 0.052^{*}$ \\
RBM & $-1.40 \pm 0.670$ & $3.40 \pm 0.330$ & $0.359 \pm 0.023^{*}$ \\
Quercetin & $3.67 \pm 0.580$ & $6.81 \pm 0.270$ & $0.403 \pm 0.063^{*}$ \\
$\begin{array}{l}\text { Alendronate } \\
\text { RBM + }\end{array}$ & $3.08 \pm 0.480$ & $7.19 \pm 0.600$ & $0.430 \pm 0.041^{*}$ \\
$\begin{array}{l}\text { Quercetin } \\
\text { RBM+ } \\
\text { Alendronate }\end{array}$ & $-1.40 \pm 0.670$ & $5.39 \pm 0.580$ & $0.393 \pm 0.021^{*}$ \\
\hline
\end{tabular}

a Three groups of rats (RBM, RBM+Quercetin, RBM+Alendronate) were administered ig with retinoic acid suspension $(80 \mathrm{mg} / \mathrm{kg} \mathrm{BW})$ once daily for 1-14 experimental days. Groups Control, Quercetin and Alendronate were treated ig with physiological solution during 1-14 days. Groups Quercetin and RBM+Quercetin were administered ig with quercetin $(100 \mathrm{mg} / \mathrm{kg} \mathrm{BW})$ once daily during 15-28 days of the study. Groups Alendronate and RBM+Alendronate were administered ig alendronate (40 mg/kg BW) once daily during 15-28 days of the study.

The 1-14 days BW change (\%) was calculated as a percentage change in body weight during 1-14 days in relation to the initial BW, and the 15-28 days BW change was calculated as a percentage of the BW change after treating three groups with retinoic acid.

${ }^{\mathrm{b}}$ Bone weight index was calculated as: Bone weight index $(\mathrm{g} / 100)=$ Right femur weight x100/Final body weight.

Number of rats per group: 7-10. Data were analyzed by Kruskal-Wallis ANOVA test. *Statistically significantly different compared to RBM $\left({ }^{*} P<0.05\right)$. ${ }^{*}$ Statistically significantly different compared to control $(\bullet P<0.05)$

Abbreviations: 13cRA - 13 cis-Retinoic acid; RBM - 13cRA-induced bone loss model.

\section{Statistical analyses}

The experiments were performed in duplicate. The results were expressed as mean \pm SEM of the representative experiment from two independent experiments. All data were analyzed by Kruskal-Wallis ANOVA. Further analysis of the differences between the groups was made with multiple comparisons of mean ranks for all groups. Statistical analyses were performed using STATISTICA 12 software (StatSoft, Tulsa, OK, USA). The data were considered significant at $P<0.05$.

\section{RESULTS}

\section{Changes in the body weight}

The initial body weights of the rats were similar between groups. Table 1 shows the mean change in body weight of both control and experimental groups after two weeks of $13 \mathrm{cRA}$ treatments and treatment of osteoporotic animals with quercetin or alendronate (four weeks). Intragastric administration of retinoic acid to rats caused a slight reduction (by $1.4 \%$ ) in body weight after 2 weeks. After a slight initial decline, the increase in body weight was observed in all groups of a minimum 3.4 to $7.19 \%$. No significant difference in body weight was found among each group.

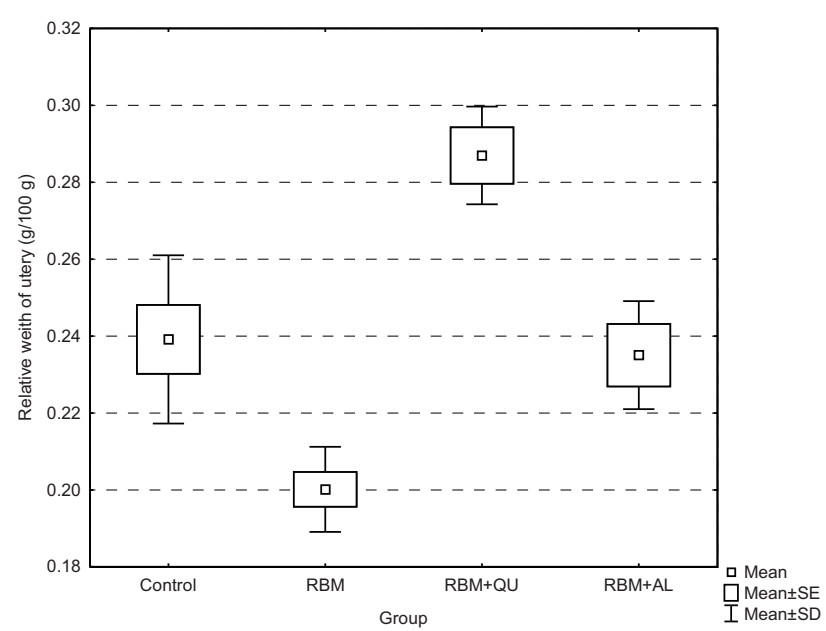

FIGURE 2. Effects of quercetin or alendronate on the changes in uterine weight index in non-osteoporotic rats and rats with retinoic acid-induced bone loss. Three groups of rats (RBM, RBM+Quercetin, $\mathrm{RBM}+$ Alendronate) were administered ig with retinoic acid suspension ( $80 \mathrm{mg} / \mathrm{kg} \mathrm{BW}$ ) once daily for 1-14 experimental days. Groups Control, Quercetin and Alendronate were treated ig with physiological solution during 1-14 days. Groups Quercetin and RBM+Quercetin were administered ig with quercetin ( $100 \mathrm{mg} / \mathrm{kg} \mathrm{BW}$ ) once daily during 15-28 days of the study. Groups Alendronate and RBM+Alendronate were administered ig alendronate $(40 \mathrm{mg} / \mathrm{kg} \mathrm{BW})$ once daily during $15-28$ days of the study. Number of rats per group: 7-10. A statistically significant difference exists between: RBM vs RBM+QU $(P<0.05)$. Abbreviations: $13 \mathrm{cRA}-13$ cis-Retinoic acid; BW - body weight; RBM - 13cRA-induced bone loss model.

\section{Changes in the uterine weight}

As shown in Figure 2, the uterine weight of RBM rats decreased from that of the control but not statistical significant. The uterine weight in the osteoporotic animals treated with QU increased significantly $(P<0.05)$ in relation to the RBM group.

In healthy animals, quercetin or alendronate treatment did not cause a significant change in uterine weight (data not shown).

\section{Physical parameters of bone and contents of calcium and phosphorus}

Femur weight and bone weight index did not differ significantly in any of the groups treated with test components and healthy control, except in RBM group $(P<0.05)$ (Table 1 and 2). The bone length and both diameters (anterior-posterior and medial-lateral) of femur in RBM group were lower than in the control group but the difference was not statistically significant (Table 2). The total contents of $\mathrm{Ca}$ and $\mathrm{P}$ in the femur of $13 \mathrm{cRA}$ treated group decreased $(P<0.05$, $P<0.05)$ compared to the control group or other experimental groups (Table 3 ). There were no statistically significant differences in $\mathrm{Ca}$, $\mathrm{P}$, bone weight, bone weight index, bone length and diameter between the control and osteoporotic groups treated with quercetin, alendronate or healthy animals treated with quercetin or alendronate (Tables 1, 2 and 3).

\section{Bone mineral density (BMD) of femur and serum levels of bone turnover markers}

The values of BMC and BMD in the proximal and distal part of the femoral neck were lower in RBM group than in the control group. Quercetin and alendronate caused a sig- 
TABLE 2. Effects of quercetin or alendronate on the body weight, bone weight, the length and the diameter of the femur bone in non-osteoporotic rats and rats with retinoic acid-induced bone loss.

\begin{tabular}{l|c|c|c|c|c|c}
\hline \multirow{2}{*}{ Treatment } & \multicolumn{6}{c}{ Measured parameter (Mean \pm SEM $)$} \\
\cline { 2 - 7 } & $\mathrm{m}_{\mathrm{rat}}(\mathrm{g})$ & $\mathrm{m}_{\text {femur- }}(\mathrm{mg})$ & $\mathrm{m}_{\text {femur-2 }}(\mathrm{mg})$ & Length of femur $(\mathrm{cm})$ & $\mathrm{R}_{\text {lat } / \mathrm{med}}(\mathrm{mm})$ & $\mathrm{R}_{\text {post/ant }}(\mathrm{mm})$ \\
\hline Control & $186 \pm 6.28$ & $733 \pm 43.2^{*}$ & $793 \pm 61.9^{*}$ & $3.18 \pm 0.030$ & $3.50 \pm 0.100$ & $3.00 \pm 0.004$ \\
RBM & $180 \pm 9.34$ & $647 \pm 23.5^{*}$ & $642 \pm 23.9^{*}$ & $3.00 \pm 0.010$ & $3.46 \pm 0.150$ & $3.22 \pm 0.180$ \\
Quercetin & $180 \pm 5.52$ & $721 \pm 78.1^{*}$ & $704 \pm 48.2^{*}$ & $3.16 \pm 0.020$ & $3.75 \pm 0.100$ & $3.06 \pm 0.060$ \\
Alendronate & $176 \pm 7.50$ & $750 \pm 42.5^{*}$ & $734 \pm 39.8^{*}$ & $3.27 \pm 0.020$ & $3.58 \pm 0.170$ & $3.08 \pm 0.080$ \\
RBM + Quercetin & $177 \pm 4.77$ & $692 \pm 27.9^{*}$ & $677 \pm 15.4^{*}$ & $3.19 \pm 0.020$ & $3.60 \pm 0.060$ & $2.85 \pm 0.060$ \\
RBM+Alendronate & $177 \pm 14.0$ & $712 \pm 24.1^{*}$ & $682 \pm 17.8^{*}$ & $3.28 \pm 0.080$ & $3.45 \pm 0.180$ & $3.27 \pm 0.270$ \\
\hline
\end{tabular}

${ }^{\text {a } T r e a t m e n t s ~ d e t a i l s: ~ s e e ~ T a b l e ~ 1 . ~ N u m b e r ~ o f ~ r a t s ~ p e r ~ g r o u p: ~ 7-10 . ~ D a t a ~ w e r e ~ a n a l y s e d ~ b y ~ K r u s k a l-W a l l i s ~ A N O V A ~ t e s t . ~ * ~ S t a t i s t i c a l l y ~ s i g n i f i c a n t l y ~ d i f f e r-~}$ ent compared to RBM $(* P<0.05)$. Statistically significantly different compared to control $(\bullet P<0.05)$.

Abbreviations: $13 \mathrm{cRA}-13$ cis-Retinoic acid; RBM - 13cRA-induced bone loss model; $\mathrm{m}_{\mathrm{rat}}-$ mean of rat body weight; $\mathrm{m}_{\mathrm{femur}-1}$ and $\mathrm{m}_{\text {femur-2}}-\mathrm{mean}$ of the right and left femurs; $\mathrm{R}_{\text {lat/med }}$ and $\mathrm{R}_{\text {post/ant }}$-the proximal epiphyseal diameters of the femur in medio-lateral and anterior-posterior directions.

TABLE 3. Effects of quercetin or alendronate on the levels of calcium (Ca) and phosphorus (P) in non-osteoporotic rats and rats with retinoic acid-induced bone loss.

\begin{tabular}{lccc}
\hline Treatment & & $\begin{array}{c}\text { mg Ca /g of wet } \\
\text { right femurs weight } \\
\text { (Mean } \pm \text { SEM) }\end{array}$ & $\begin{array}{c}\text { mg P/g of wet right } \\
\text { femurs weight } \\
\text { (Mean } \pm \text { SEM) }\end{array}$ \\
\hline Control & $135 \pm 3.59^{*}$ & $57.3 \pm 1.25^{*}$ \\
RBM & $121 \pm 3.30^{*}$ & $49.3 \pm 2.15^{*}$ \\
Quercetin & $143 \pm 6.28^{*}$ & $59.3 \pm 2.02^{*}$ \\
Alendronate & $134 \pm 5.94^{*}$ & $54.7 \pm 4.98^{*}$ \\
RBM + Quercetin & $139 \pm 5.04^{*}$ & $57.4 \pm 1.21^{*}$ \\
RBM+ Alendronate & $136 \pm 5.17^{*}$ & $61.0 \pm 2.30^{*}$ \\
\hline
\end{tabular}

a Treatments details: see Table 1. Number of rats per group: 7-10. Results are expressed as mg calcium $(\mathrm{Ca})$ and phosphorus $(\mathrm{P})$ per gram of wet right femurs weight. Data were analysed by Kruskal-Wallis ANOVA test. * Statistically significantly different compared to RBM $(* P<0.05)$. ${ }^{*}$ Statistically significantly different compared to control $(\bullet P<0.05)$.

Abbreviations: 13cRA - 13 cis-Retinoic acid; RBM - 13cRA-induced bone loss model.

nificant increase in BMC and BMD values in the proximal $(P<0.05, P<0.05)$ part and alendronate in the distal $(P<0.05)$ part of the femoral neck (Figure 3A) in relation to RBM. Application of quercetin or alendronate did not lead to changes in the value of BMD in proximal and distal region in healthy rats compared to controls (data not shown).

Serum $\beta$-CTx concentration was significantly increased in the RBM group $(P<0.05)$ when compared with healthy control (Figure 3B). Interestingly, serum osteocalcin (OC) concentration was also reduced by RBM while treatment of osteoporetic mice with QU significantly $(P<0.05)$ increased OC when compared with RBM.

\section{Histological changes in bone}

Histological analysis of the distal femur diaphysis revealed that in control rats its cortex consisted primarily of circumferen- tial lamellar bone, which contained only cellular lamellae and osteocytes inside round or oval lacunae (Figure 4A, C, D). Haversian canals have been observed in the middle part of the bone tissue (Figure 4C, D) particularly at the anterior side of the section. Few small marrow-filled intracortical cavities were also seen (Figure 4A, B) as well as remnants of woven bone (Fig 4D). Periosteum and endosteum were smooth with no irregularities.

Compared with the control group, diaphyseal cortex of rats treated with 13cRA showed thinning accompanied with marked porosity caused by formation of multiple cavities of different sizes (Figure 4E) while endosteal surface appeared irregular with many notches (Figure 4G). At the cortical surface, cavities were bounded with osteoid or newly formed bone, while luminal surface was lined with osteoblasts and osteoclasts (Figure. 4F). Osteoclasts were also observed housed in the irregularly eroded endosteal surface (Figure 4G).

Diaphyseal cortex of osteoporotic rats treated with alendronate showed signs of improvement of bone structure (Figure $4 \mathrm{H}$ ). The thickness of cortical bone increased, osteoporotic cavities were reduced in their number and size but endosteal surface still showed an irregularity at many places (Figure 4H). Osteoporotic rats treated with quercetin showed marked improvement of the structure of femoral cortical bone, which showed thickness nearly similar to that of the control group although a few small intracortical cavities were still present (Figure 4I). Moreover, endosteal surface became smooth with few slight irregularities.

\section{Changes in biochemical and hematological parameters}

Treatment with retinoic acid resulted in a significant increase in ALP and serum total protein $(P<0.05 ; P<0.05)$ compared to control (Table 4).

The oral administration of quercetin or alendronate via a gavage needle to osteoporotic rats caused a significant decrease in ALP and LDH levels $(P<0.05 ; P<0.05)$ in relation to RBM.

Results indicated that RBM and other test compounds caused a significant increase in total leukocyte count $(P<0.05)$ in relation to control while $\mathrm{QU}$ and alendronate caused a decrease in the platelet count $(P<0.05)$ in healthy animals com- 
A
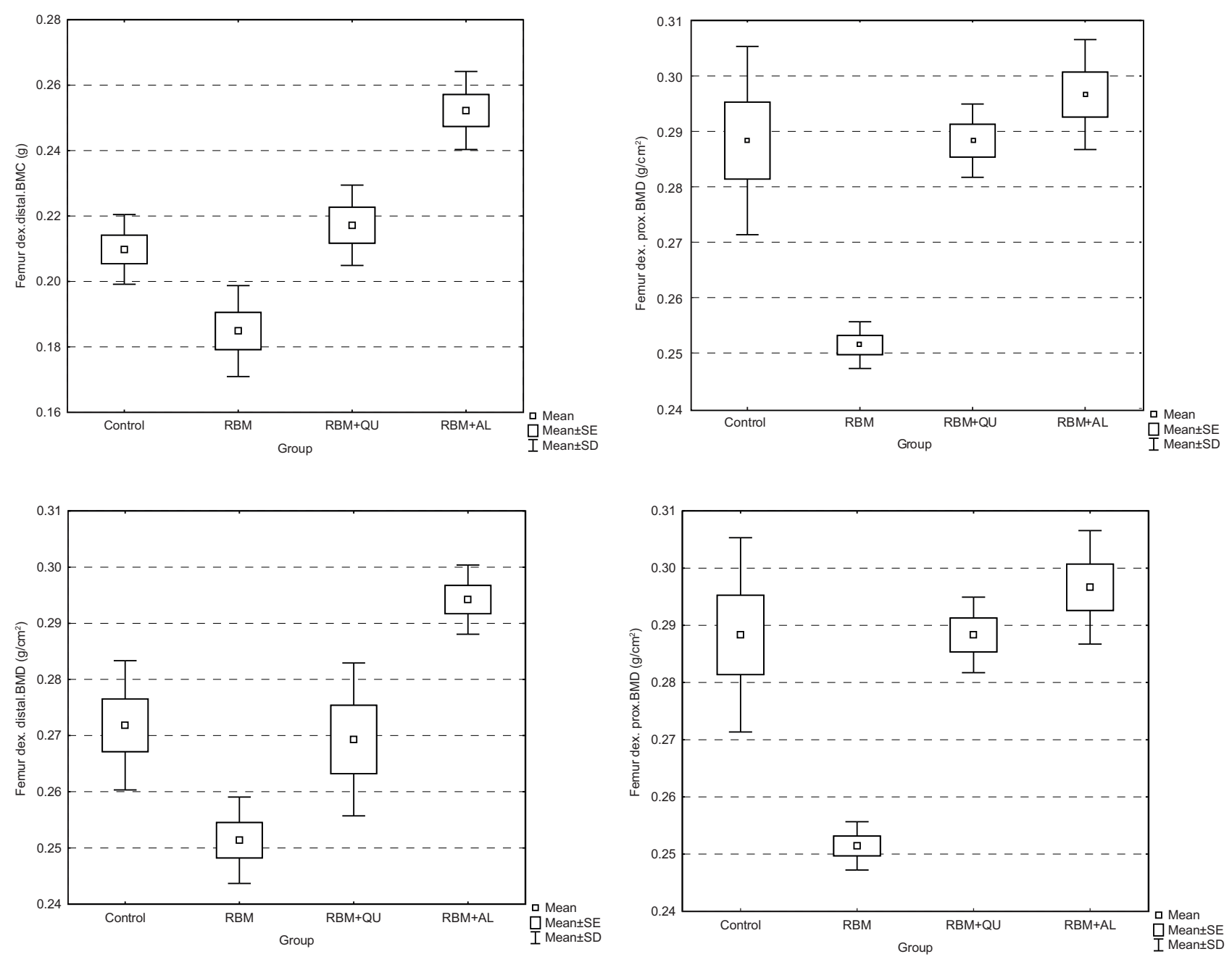

B
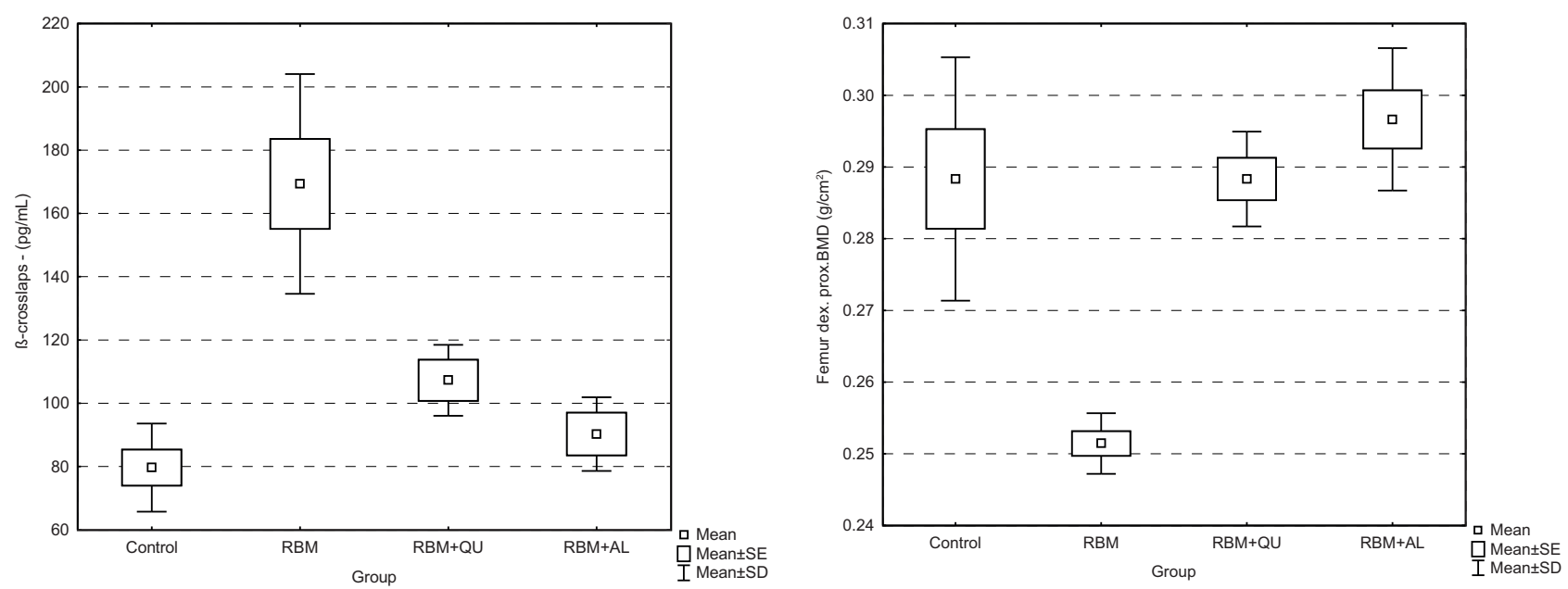

FIGURE 3. Effects of quercetin or alendronate on the bone mineral density (A) and serum markers of bone turnover (B) in non-osteoporotic rats and rats with retinoic acid-induced bone loss. Three groups of rats (RBM, RBM+Quercetin, RBM+Alendronate) were administered ig with retinoic acid suspension ( $80 \mathrm{mg} / \mathrm{kg} \mathrm{BW}$ ) once daily for 1-14 experimental days. Groups Control, Quercetin and Alendronate were treated $i g$ with physiological solution during 1-14 days. Groups Quercetin and RBM+Quercetin were administered ig with quercetin (100 mg/kg BW) once daily during 15-28 days of the study. Groups Alendronate and RBM+Alendronate were administered ig alendronate $(40 \mathrm{mg} / \mathrm{kg} \mathrm{BW})$ once daily during $15-28$ days of the study. Number of rats per group: 7-10. (A) A statistically significant difference exists between: Prox, RBM $v s$ RBM+QU $(P<0.05)$, RBM $v s$ RBM + AL $(P<0.05)$; Dist, RBM vs RBM + AL $(P<0.05)$. (B) A statistically significant difference exists between: $\beta$-CTx, Control vs RBM $(P<0.05)$; OC, RBM vs RBM+QU $(P<0.05)$. Abbreviations: 13cRA - 13 cis-Retinoic acid; BW - body weight; RBM - 13cRA-induced bone loss model; BMC - bone mineral content; BMD - bone mineral density; OC - osteocalcin; $\beta$-CTx - $\beta$-CrossLaps. 



FIGURE 4. Cross sections of the distal femur diaphysis cortical bone of female rats stained with HE. (A-D) Control group. (A) Cortical bone showing normal architecture of the bone tissue. (B-D) Higher magnification of section (a) showing cortex with inner and outer circumferential bone tissue (C, D) containing cellular lamellae and osteoclasts inside round or more oval lacunae. Haversian canals (Hc) are seen in the middle part of the bone tissue (C, D) with slightly higher density at the anterior side of the section (B). Few small intracortical cavities (A, B) are also seen as well as remnants of woven bone (D). (E-G) Retinoic acid-treated group (osteoporotic group). (E) Formation of multiple large intracortical cavities and thinning of the cortex. (F) Higher magnification of the highlighted area of section (e) showing intracortical cavities bounded with newly formed bone and osteoclasts lining the luminal surface of the cavities. (G) Irregular endosteum with notches containing osteoclasts. (H) Osteoporotic rats treated with alendronate. Marked improvement of the structure of the cortical bone with thickness similar to that of control group although few intracortical cavities are still present. (I) Osteoporotic rats treated with quercetin. Marked improvement of the structure of cortical bone which showed a few small intracortical cavities and thickness nearly similar to that of control group. Abbreviations: 13cRA - 13 cis-Retinoic acid; BW - body weight; RBM - 13cRA-induced bone loss model. 
TABLE 4. Effects of quercetin or alendronate on serum biochemical parameters in non-osteoporotic rats and rats with retinoic acid-induced bone loss.

\begin{tabular}{l|c|c|c|c|c|c|c}
\hline \multirow{2}{*}{ Treatment } & \multicolumn{7}{c}{ Biochemical values (Mean \pm SEM) } \\
\cline { 2 - 8 } & ALP (U/L) & AST (U/L) & ALT (U/L) & LDH (U/L) & TP (g/L) & GLU (mmol/L) & Urea (mmol/L) \\
\hline Control & $168 \pm 3.91^{*}$ & $89.0 \pm 8.15$ & $46.4 \pm 4.76$ & $611 \pm 21.2$ & $53.0 \pm 0.670^{*}$ & $6.46 \pm 0.130$ & $6.26 \pm 0.410$ \\
RBM & $206 \pm 7.97^{*}$ & $89.7 \pm 5.54$ & $43.2 \pm 1.90$ & $572 \pm 11.3$ & $56.0 \pm 0.570^{*}$ & $7.29 \pm 0.370$ & $5.65 \pm 0.320$ \\
Quercetin & $105 \pm 2.07^{* *}$ & $83.7 \pm 5.13$ & $46.6 \pm 2.10$ & $522 \pm 35.0$ & $52.8 \pm 1.14$ & $6.65 \pm 0.340$ & $5.61 \pm 0.140$ \\
Alendronate & $90.1 \pm 4.14^{* *}$ & $82.3 \pm 3.12$ & $49.5 \pm 2.03$ & $595 \pm 64.1$ & $55.4 \pm 0.490$ & $6.20 \pm 0.240$ & $6.20 \pm 0.200$ \\
RBM + Quercetin & $140 \pm 6.96^{* \bullet}$ & $77.7 \pm 2.60$ & $38.0 \pm 2.08$ & $422 \pm 34.0^{* \bullet}$ & $53.3 \pm 0.330$ & $7.95 \pm 0.520$ & $5.66 \pm 0.170$ \\
RBM + Alendronate & $103 \pm 14.1^{* \bullet}$ & $87.7 \pm 4.33$ & $42.0 \pm 1.52$ & $402 \pm 71.0^{* \bullet}$ & $51.7 \pm 2.33$ & $6.23 \pm 0.540$ & $6.76 \pm 0.210$ \\
\hline
\end{tabular}

${ }^{a}$ Treatments details: see Table 1. Number of rats per group: 7-10. Data were analysed by Kruskal-Wallis ANOVA test. * Statistically significantly different compared to RBM $\left({ }^{*} P<0.05\right)$. $\bullet$ Statistically significantly different compared to control $\left({ }^{*} P<0.05\right)$.

Abbreviations: 13cRA - 13 cis-Retinoic acid; RBM - 13cRA-induced bone loss model; AST - aspartate aminotransferase; ALT - alanine aminotransferase; ALP - alkaline phosphatase; LDH - lactate dehydrogenase; TP - total protein; GLU - glucose.

TABLE 5. Effects of quercetin or alendronate on haematological parameters in non-osteoporotic rats and rats with retinoic acid-induced bone loss.

\begin{tabular}{|c|c|c|c|c|c|c|c|c|}
\hline \multirow{2}{*}{ Treatment ${ }^{\mathrm{a}}$} & \multicolumn{8}{|c|}{ Haematological values $($ Mean \pm SEM $)$} \\
\hline & $\mathrm{L}\left(10^{9} / \mathrm{L}\right)$ & $\mathrm{E}\left(10^{12} / \mathrm{L}\right)$ & $\mathrm{Hgb}(\mathrm{g} / \mathrm{L})$ & Hct & $\mathrm{MCV}$ (fL) & $\mathrm{MCH}(\mathrm{pg})$ & $\mathrm{MCHC}(\mathrm{g} / \mathrm{L})$ & Plt $\left(10^{9} / \mathrm{L}\right)$ \\
\hline Control & $2.70 \pm 0.590^{*}$ & $7.29 \pm 0.230$ & $145 \pm 6.49$ & $0.440 \pm 0.010$ & $60.1 \pm 0.780$ & $20.0 \pm 0.450$ & $333 \pm 4.65$ & $729 \pm 38.6$ \\
\hline RBM & $3.88 \pm 0.460^{\star}$ & $6.88 \pm 0.120$ & $132 \pm 2.07$ & $0.400 \pm 0.005$ & $56.8 \pm 1.41$ & $19.2 \pm 0.120$ & $329 \pm 2.30$ & $722 \pm 22.6$ \\
\hline Quercetin & $5.62 \pm 0.200^{* \star}$ & $7.48 \pm 0.160$ & $134 \pm 1.26$ & $0.430 \pm 0.004$ & $58.1 \pm 0.620$ & $17.8 \pm 0.280$ & $307 \pm 2.10$ & $460 \pm 17.9^{* \bullet}$ \\
\hline Alendronate & $5.12 \pm 0.240^{* \bullet}$ & $6.92 \pm 0.240$ & $130 \pm 1.76$ & $0.420 \pm 0.004$ & $61.5 \pm 1.67$ & $18.9 \pm 0.050$ & $307 \pm 0.860$ & $475 \pm 24.7^{* \bullet}$ \\
\hline $\begin{array}{l}\text { RBM+ } \\
\text { Quercetin }\end{array}$ & $4.50 \pm 0.340^{\star}$ & $6.91 \pm 0.190$ & $130 \pm 3.21$ & $0.390 \pm 0.010$ & $57.7 \pm 0.200$ & $18.9 \pm 0.140$ & $327 \pm 3.48$ & $666 \pm 14.1^{* *}$ \\
\hline $\begin{array}{l}\text { RBM } \\
+ \text { Alendronate }\end{array}$ & $4.70 \pm 0.430^{\circ}$ & $6.57 \pm 0.200$ & $128 \pm 3.78$ & $0.380 \pm 0.007$ & $58.9 \pm 0.710$ & $19.3 \pm 0.130$ & $328 \pm 3.46$ & $749 \pm 16.3$ \\
\hline
\end{tabular}

${ }^{a}$ Treatments details: see Table 1. Number of rats per group: 7-10. Data were analysed by Kruskal-Wallis ANOVA test. * Statistically significantly different compared to RBM $\left({ }^{*} P<0.05\right)$. $*$ Statistically significantly different compared to control $(* P<0.05)$.

Abbreviations: 13cRA - 13 cis-Retinoic acid; RBM - 13cRA-induced bone loss model; L - leukocytes; E - erythrocytes; Hgb - hemoglobin; HCT hematocrit; MCV- average volume of red blood cells; $\mathrm{MCH}$ - the average amount of hemoglobin in red blood cells; MCHC - mean concentration of hemoglobin in red blood cells; Plt - platelets.

pared to control (Table 5). Treatment of osteoporotic rats with quercetin caused a significant $(P<0.05)$ decrease in platelet count in relation to healthy control, or animals treated with $13 \mathrm{cRA}$.

\section{Oxidative and antioxidative activity in liver, kidney and ovary}

Results of oxidative stress and antioxidative enzyme activity in osteoporotic rats after treatment with QU or alendronate are depicted in Table 6.

Table 6 clearly shows that the 13cRA (RBM model) in all organs, except spleen, caused a significant increase in the level of MDA $(P<0.05)$ and reduced GSH level $(P<0.05)$. There was no statistically significant difference between the MDA and GSH values in the kidney, liver and ovaries in the osteoporotic animals treated with quercetin compared to healthy controls. Treatment of rats with alendronate increased the level of MDA in all organs, showing increased toxicity accompanied by a compensatory increase in GSH and CAT activity.
In healthy animals, quercetin or alendronate treatment did not cause a significant change in MDA, GSH, SOD or CAT activity in liver, kidney, and ovary compared to control (data not shown).

\section{DISCUSSION}

According to our data and the data of other authors [Fahmy \& Soliman, 2009; Oršolić et al., 2014; Wei et al., 2007], the main effects of 13cRA that contribute to bone loss are: a) increased oxidative stress (OS) and the formation of reactive radicals, decreased activity of osteoblasts and increased activity of osteoclast; b) osteoblasts apoptosis; c) decreased levels of estrogen and appearance of inflammatory cytokines, d) reduced activity of vitamin D receptor, decreased absorption of $\mathrm{Ca}^{2+}$ in the intestine, increased excretion of calcium through the kidneys; and d) the effects on parathyroid hormone.

Based on the facts mentioned above this model of osteoporosis caused by $13 \mathrm{cRA}$ is the ideal model for studying anti- 
TABLE 6. Effects of quercetin or alendronate on MDA and GSH contents and SOD and CAT activity in ovary, kidney, spleen and liver in non-osteoporotic rats and rats with retinoic acid-induced bone loss.

\begin{tabular}{|c|c|c|c|c|}
\hline \multirow{2}{*}{ Treatment $^{\mathrm{a}}$} & \multicolumn{4}{|c|}{ MDA level (Mean \pm SEM) (nmol/mg protein) } \\
\hline & Ovary & Kidney & Spleen & Liver \\
\hline Control & $12.1 \pm 1.62 *$ & $4.10 \pm 0.595^{*}$ & $16.2 \pm 1.05$ & $10.2 \pm 0.559^{*}$ \\
\hline RBM & $35.8 \pm 3.45^{\bullet}$ & $11.5 \pm 1.26^{\bullet}$ & $17.6 \pm 1.56$ & $14.1 \pm 0.625^{\bullet}$ \\
\hline RBM+ Quercetin & $12.2 \pm 3.93$ & $5.10 \pm 1.00$ & $12.9 \pm 2.06$ & $9.90 \pm 1.26$ \\
\hline \multirow[t]{2}{*}{ RBM + Alendronate } & $43.9 \pm 9.77^{\bullet}$ & $9.80 \pm 1.38$ & $23.6 \pm 2.15$ & $18.1 \pm 1.33$ \\
\hline & \multicolumn{4}{|c|}{ Total GSH level (Mean \pm SEM) ( $\mu \mathrm{g} / \mathrm{mg}$ protein) } \\
\hline Control & $0.960 \pm 0.154$ & $8.45 \pm 0.114$ & $14.8 \pm 2.25$ & $8.90 \pm 0.407$ \\
\hline RBM & $0.200 \pm 0.016^{\bullet}$ & $3.60 \pm 0.322^{\bullet}$ & $8.40 \pm 0.375$ & $5.90 \pm 0.374^{\bullet}$ \\
\hline RBM+ Quercetin & $1.01 \pm 0.146^{*}$ & $6.30 \pm 0.631$ & $5.80 \pm 0.132^{*}$ & $7.00 \pm 1.34$ \\
\hline \multirow[t]{2}{*}{ RBM + Alendronate } & $0.370 \pm 0.195$ & $10.6 \pm 0.607^{\bullet}$ & $12.7 \pm 1.27$ & $5.10 \pm 1.20$ \\
\hline & \multicolumn{4}{|c|}{ SOD activity (Mean \pm SEM) (U/mg protein) } \\
\hline Control & $15.5 \pm 1.81$ & $4.10 \pm 0.478$ & $5.37 \pm 0.521$ & $3.70 \pm 0.686$ \\
\hline RBM & $7.43 \pm 0.687$ & $3.90 \pm 0.522$ & $4.22 \pm 1.14$ & $1.70 \pm 0.131^{\bullet}$ \\
\hline RBM+ Quercetin & $3.13 \pm 0.713^{\bullet}$ & $4.60 \pm 0.581$ & $3.38 \pm 1.32$ & $1.89 \pm 0.372$ \\
\hline \multirow[t]{2}{*}{ RBM + Alendronate } & $2.46 \pm 0.428^{\bullet}$ & $7.74 \pm 1.75$ & $5.28 \pm 1.37$ & $1.76 \pm 0.206$ \\
\hline & \multicolumn{4}{|c|}{ CAT activity (Mean \pm SEM) $(\mathrm{U} / \mathrm{mg}$ protein $)$} \\
\hline Control & $0.900 \pm 0.061$ & $22.0 \pm 1.58$ & $4.20 \pm 0.280$ & $5.00 \pm 0.418$ \\
\hline RBM & $11.6 \pm 1.73$ & $8.90 \pm 0.727$ & $2.80 \pm 0.400$ & $4.70 \pm 0.611$ \\
\hline RBM+ Quercetin & $19.8 \pm 0.868^{\bullet}$ & $55.9 \pm 5.67^{*}$ & $2.90 \pm 1.16$ & $3.40 \pm 0.325$ \\
\hline RBM + Alendronate & $20.9 \pm 2.10^{\bullet}$ & $66.4 \pm 4.21^{*}$ & $7.00 \pm 0.563^{*}$ & $4.10 \pm 0.860$ \\
\hline
\end{tabular}

a Three groups of rats (RBM, RBM+Quercetin, RBM+Alendronate) were administered ig with retinoic acid suspension ( $80 \mathrm{mg} / \mathrm{kg}$ BW) once daily for 1-14 experimental days. Group Control was treated ig with physiological solution during 1-14 days. Group RBM+Quercetin was administered $i g$ with quercetin ( $100 \mathrm{mg} / \mathrm{kg} \mathrm{BW}$ ) once daily during 15-28 days of the study. Group RBM+Alendronate was administered ig alendronate (40 mg/kg BW) once daily during 15-28 days of the study.

Number of rats per group: 7-10. Data were analysed by Kruskal-Wallis ANOVA test. * Statistically significantly different compared to RBM (*P<0.05). -Statistically significantly different compared to control $(\bullet \mathrm{P}<0.05)$.

Abbreviations: 13cRA - 13 cis-Retinoic acid; RBM - 13cRA-induced bone loss model; MDA - malondialdehyde; GSH - glutathione; SOD - superoxide dismutase; CAT - catalase.

oxidative, anti-inflammatory, phytoestrogenic, and regenerative capacity of flavonoid quercetin on 13cRA-induced bone loss model.

Osteoporosis in this model is similar to human in many ways, such as symptoms, histomorphology and ossis response to estrogenic [Peng et al., 2005]. Thus, 13cRA is an ideal agent to induce acute osteoporosis model. An increasing amount of clinical evidence suggests a role for the dietary polyphenols and nutritional supplements in the treatment of postmenopausal bone loss [Coxam, 2008]. In order to clarify this issue, we investigated the therapeutic effects of quercetin compared with alendronate in an experimental animal model of RBM. Effect of quercetin on bone metabolism was assessed by monitoring several parameters such as the changes in mass of experimental animals, uterine weight, femur weight, length and the diameter of the femur, BMD, markers of osteoporosis, and histopathological analysis. In addition, we also analyzed the content of calcium and phosphorus after carbonization of bone and biochemical and hematological variables with special reference to changes in serum ALP. The analysis was carried out in two steps.

In the first step of our experiment we have revaluated the osteoporotic potential of 13cRA and we have attempted to assess the effectiveness of the flavonoid quercetin in reversing bone loss. Retinoic acid successfully induced osteoporoticlike changes; these changes are confirmed by reduced values of BMD in the proximal and distal part of the femoral neck (Figure 3), geometrical and physical parameters of bone, content of $\mathrm{Ca}$ and $\mathrm{P}$ in bones as well as in bone turnover markers (Tables 1, 2 and 3).

Our results of histological analysis of femoral cortical bone revealed that 13cRA exerted pathological changes characterized by the presence of multiple osteoporotic cavities of different sizes and thinning of the bone cortex which 
is consistent with the significant decrease in femur bone mineral density and both calcium and phosphorous content (Figure 4). As we also noticed increase in osteoclast number on the luminal surface of cavities and irregularly eroded endosteal surface, this bone loss may be due to increased osteoclastic activity and consequently increase in bone resorption. Receptors for $13 \mathrm{cRA}$ are found on both bone cells osteoblasts and osteoclasts, which indicate that they are direct targets of 13cRA [Rohde et al., 1999]. Previous in vitro studies have reported that $13 \mathrm{cRA}$ stimulates osteoclast formation and suppresses osteoblast activity [Togari et al., 1991], but recently [Peng et al., 2005] showed that bone resorption exceeded bone formation due to the promotion effect of $13 \mathrm{cRA}$ on osteoclasts instead of osteoblasts inhibition. This was supported by the phenomena that 13cRA-treated group showed as active proliferation of osteoblasts as the control and a marked increase in osteoclast number and activity. Our results from the qualitative histological analysis also showed that $13 \mathrm{cRA}$ stimulates both bone cells. We found that resorption cavities were bounded with osteoid or newly formed bone showing that bone formation followed bone resorption. This also showed that $13 \mathrm{cRA}$ has a more pronounced effect on osteoclasts, which results in an unbalance between bone resorption and formation, and leads to a bone loss (Figure 3 ). The increase in osteoclasts is associated with oxidative stress which was confirmed by many authors [Fahmy \& Soliman, 2009; Oršolić et al., 2014; Togari et al., 1991; Walker-Bone, 2012]. Our results demonstrate that 13cRA induces increased oxidative stress by increasing the levels of MDA and reducing the levels of glutathione and antioxidant enzymes in the liver and kidney and ovary (Table 6).

Some authors have shown that high doses of $13 \mathrm{cRA}$ cause osteoporosis, reduce BMD and increase bone fragility [Kneissel et al., 2005]. Our results also proved increased bone turnover rate as indicated by the higher serum ALP level in the RBM group compared to the control group. Apart from ALP, the treatment with RBM resulted in a significant increase in ALT, and serum total protein (TP) and leukocyte count compared to control. These results revealed a low level of toxicity of $13 \mathrm{cRA}$ on bone and liver cells. These results are in agreement with findings reported by other authors [Fahmy \& Soliman, 2009; Mukherjee et al., 2004; Wei et al., 2007], who demonstrated that bone remodeling in rats is accelerated after ovariectomy-induced model of osteoporosis. These results are consistent with the data from Hotchkiss et al. [2006] who showed that retinoids in large doses cause an increase of periosteal resorption in relation to periosteal extension or disposal of minerals, which ultimately leads to the reduction in the diameter of bone mineral loss. This decrease may be due to the antagonizing effect of 13cRA on vitamin D as suggested by Johansson \& Melhus [2001] who proved the effectiveness of retinoic models for osteoporosis by applying 13cRA at a dose of $80 \mathrm{mg} / \mathrm{kg}$ for 2 weeks that caused osteoporotic changes.

Our result suggests that quercetin may have protective effects against 13cRA-induced bone resorption. The use of quercetin in treatment of osteoporosis showed improvement of femoral cortical bone histology, BMD, bone weight, length and diameters as well as $\mathrm{Ca}$ and P (Figure 3, Tables 1, 2 and 3 ). These results suggest that 13cRA-induced increase in bone resorption could be blocked by quercetin as an inhibitor of osteoclast activity (Figure 3A, B). Furthermore, treatment of osteoporetic rats with QU significantly increased osteocalcin (OC) concentrations when compared with RBM.

This assumption is consistent with the results of Wattel et al. [2004] and Woo et al. [2004] who investigated the effects of quercetin on osteoclast differentiation, which is a crucial determination step of in vivo bone resorption, and found that quercetin suppresses bone resorption by inhibiting both, the differentiation and activation of osteoclasts. It can also directly induce apoptosis of mature osteoclasts [Wattel et al., 2004].

Quercetin-treated RBM rats were superior to the untreated rats in the levels of $\mathrm{Ca}$ and $\mathrm{P}(P<0.05, P<0.05)$. Ca and $\mathrm{P}$ are widely accepted phenotype markers of the bone formation. In addition, treatments of osteoporotic animals with quercetin resulted in an increase in body mass by $5.32 \%$ after four weeks. Antiosteoporotic activity of flavonoids, including QU, is based on osteoblast stimulation mediated by estrogen receptors and inhibition of osteoclast as well as antioxidative and anti-inflammatory activity [Prouillet et al., 2004; Wattel et al., 2004; Xu et al. 2012]. Others studies show that dietary quercetin as well as other flavonoids can inhibit bone loss in ovariectomized mice [Tsuji et al., 2009] and in rat model of diabetic osteopenia [Liang et al., 2011], confirming the beneficial effect of quercetin on bone tissue in bone disorders. In addition, quercetin consumption may improve calcium absorption in small intestine and enhance VDR (Vitamin D receptor) activity that regulates the expression of genes involved in calcium homeostasis. Activation of VDR is thought to be a promising drug target for osteoporosis [Inoue et al., 2010]. According to our data, it is evident that quercetin beneficial effect on bone is achieved through its antioxidant and anti-inflammatory abilities; quercetin successfully inhibited 13cRA-induced OS (Figure 5).

Our study indicates that a decreased glutathione level and an increased MDA level in RBM group is related to increased ROS production and that supplementation with quercetin significantly reduced oxidative stress parameters in osteoporotic groups. In addition, recent evidences implicate reactive oxygen species (ROS) in estrogen deficiency-induced bone loss [Sheweita \& Khoshhal, 2007] and enhanced osteoclastic and depressed osteoblastic activity in primary osteoporosis in post-menopausal women. Retinoic acid was found to decrease estrogen level in blood [Muthusami et al., 2005]. Indeed, 13cRA in our experiment resulted in a decrease in uterine weight (Figure 2) and GSH level in ovary while the level of MDA in ovary was increased. Since both QU and AL increase uterine weight, it is suggested that these agents influence the actions of estrogen or its receptor. QU induced better estrogenic effect; uterine weight was higher compared to the control group. In addition, QU reduces the level of MDA in the ovaries and thus directly increases estrogen activity. Indeed, estrogen has been shown to have antioxidant properties [Fahmy \& Soliman, 2009; Prouillet et al., 2004].

These results suggest that a daily supplementation with quercetin may be important in reducing oxidative damage of 13cRA and other drugs which leads to extensive bone loss and skeletal fragility, characteristic of osteoporosis. In addi- 
tion, we propose that the antioxidant properties of quercetin may prevent the free-radical-induced bone loss associated with estrogen deficiency.

In addition, there was no significant increase in the value of liver enzymes after administration of quercetin. Results also revealed more pronounced increase in leukocyte values after administration of quercetin, which is in conformance with the data from the literature [Oršolić et al., 2010, 2011] based on immunomodulatory effect of quercetin. From these results we can clearly conclude that quercetin had no significant toxic effects on experimental animals.

These results are similar to findings of Mackinnon et al. [2011] who investigated the effectiveness of lycopene, a fat-soluble antioxidant, on oxidative stress parameters in humans. Results of their study showed a decrease in oxidized proteins and lipid peroxidation at 4 weeks of treatment with lycopene.

Many authors demonstrated that diet supplemented with antioxidants like vitamins $\mathrm{C}, \mathrm{E}$ and flavonoids could prevent bone loss in osteoporosis [Bitto et al., 2009; Coxam, 2008; Iwaniec et al., 2013; Oršolić et al., 2014; Wei et al., 2007]. On the other hand, deficiency in vitamins E and D may cause a decrease in cartilage cells and osteocytes, as well as thinning of the cortex and trabecular in mice [Iwaniec et al., 2013].

In turn, treatments of osteoporotic animals with alendronate resulted in an increase in body mass by $3.94 \%$ and significant improvement in calcium and phosphorus levels in bone histology and BMD after four weeks (Table 2, Figure 3).

Alendronate is a bisphosphonate drug used for the treatment of postmenopausal and other forms of osteoporosis and diseases characterized by bone loss, as many studies using a variety of experimental systems demonstrated that bisphosphonates are able to inhibit osteoclast-mediated bone resorption in various animal models [Chavassieux et al., 1997; Huuskonen et al. 2001]. Long-term treatment of human and nonhuman primates with alendronate has been shown to increase cortical thickness, an index of increased osteoblast number or activity [Chavassieux et al., 1997], raising the possibility that it may not only inhibit bone resorption, but may also have a positive effect on bone formation [Manolagas, 2009]. Huuskonen et al. [2001] examined the effect of alendronate $(18 \mu \mathrm{g} / \mathrm{kg})$ on rat femur bone following orchidectomy-induced osteoporosis and found that alendronate increased the ultimate bending force of the femoral diaphysis, histologically it reduced the trabecular bone turnover but was not able to maintain the bone mineral density of the proximal femur at the pre-orchidectomy level. Bitto et al. [2009] also showed that alendronate succeeded in treating glucocorticoid-induced osteoporosis in rats as it increased bone breaking strength, bone mineral density and ameliorated histological damage of both cortical and trabecular bone matrix in femur head, while more detailed histological and histochemical study of Ali [2006] revealed markedly reduced osteoporotic cavities, highly degenerated osteoclasts, an increase in the collagenous fibers in the periosteum and in bone matrix and positive ALP immune reaction surrounding most of bone cells.

Our results as well showed significant improvement in bone histology in alendronate-treated 13cRA-induced osteoporotic rats as we found fewer and smaller intracortical cavities (Figure 4). Despite the positive effects of alendronate on bone after 13cRA-induced osteoporosis, ALP activity, $\mathrm{BMD}, \mathrm{Ca}$ and $\mathrm{P}$ levels, alendronate has increased the level of MDA in the kidney, suggesting increased oxidative stress that can cause kidney damage during its longer administration (Table 6). Increased levels of MDA, GSH and antioxidant enzymes in the group treated with alendronate may be indirect compensatory mechanism designed to protect the kidneys from ROS-mediated insult.

The obtained data are in compliance with the data of other authors, who suggest that bisphosphonates often cause renal insufficiency and gastrointestinal adverse effects [Conwell \& Chang, 2012; Perazella \& Markowitz, 2008]. The toxic effect of bisphosphonates was demonstrated on kidney; acute tubular necrosis and focal segmental glomerulosclerosis were observed as a result of oxidative stress and mononuclear leukocyte accumulation and matrix production and degradation, respectively.

In the second step, we analyzed the effect of quercetin or alendronate in healthy rats. Obtained data suggest possibilities of quercetin use in the prevention of osteoporosis. Administration of quercetin in healthy animals resulted in a higher - though statistically insignificant - content of calcium and phosphorus than in the control group. After alendronate application, calcium and phosphorus contents were similar to these in the control group. Glutathione level in the kidney was increased in healthy rats treated with quercetin when compared to the control, while alendronate did not cause a significant change in liver in relation to control but it decreased GSH level in kidney. Perazella \& Markowitz [2008] showed similar results in their research, where they confirmed that alendronate-induced oxidative gastric damage, leading to a reduced $\mathrm{GSH}$ level in rats.

\section{CONCLUSIONS}

To summarize our findings, quercetin effectively improved bone structure and prevented bone loss in osteoporosis animal model. The beneficial effects of quercetin on bone histology, BMD, osteocalcin, bone weight, $\mathrm{Ca}$ and $\mathrm{P}$ level may be due to its antioxidant and estrogenic property. Quercetin as a strong antioxidant - can reduce the toxic effects of $13 \mathrm{cRA}$ on bone loss and sustain bone homeostasis in rats better than alendronate.

All those findings indicate the beneficial effect of quercetin on osteoporosis caused by 13cRA in rats, and raise the possibility of developing quercetin as a potential drug or an ingredient in diet for controlling drug-induced osteoporosis.

\section{HUMAN AND ANIMAL RIGHTS}

All animal experiments carried out in compliance with the Guide for the Care and Use of Laboratory Animals, DHHS Publ. \# (NIH) 86-123.

\section{ACKNOWLEDGEMENTS}

This work was supported by the Ministry of Sciences, Education and Sports of the Republic of Croatia project No. 119-0000000-1255. 


\section{CONFLICTS OF INTEREST}

Authors declare no conflict of interest.

\section{REFERENCES}

1. Ali A.A., Effect of alendronate sodium (Fosamax) on bone of adult male Sprague dawley rats under glucocorticoids therapy histological and histochemical study. Egypt. J. Histol., 2006, 29, $1,61-72$.

2. Bitto A., Burnett B.P., Polito F., Levy R.M., Marini H., Di Stefano V., Irrera N., Armbruster M.A., Minutoli L., Altavilla D., Squadrito F., Genistein aglycone reverses glucocorticoid-induced osteoporosis and increases bone breaking strength in rats: a comparative study with alendronate. Br. J. Pharmacol., 2009, 156, 1287-1295.

3. Chavassieux P.M., Arlot M.E., Reda C., Wei L., Yates A.J., Meunier P.J., Histomorphometric assessment of the long-term effects of alendronate on bone quality and remodeling in patients with osteoporosis. J. Clin. Invest., 1997, 100, 1475-1480.

4. Chiang C.H., Huang C.C., Chan W.L., Huang P.H., Chen T.J., Chung C.M., Lin S.J., Chen J.W., Leu H.B., Oral alendronate use and risk of cancer in postmenopausal women with osteoporosis: A nationwide study. J. Bone. Miner. Res., 2012, 27, 1951-1958.

5. Conwell L.S., Chang A.B., Bisphosphonates for osteoporosis in people with cystic fibrosis. Cochrane Database. Syst. Rev. 2012, doi: 10.1002/14651858.CD002010.pub3.

6. Coskun O., Kanter M., Korkmaz A., Oter S., Quercetin, a flavonoid antioxidant, prevents and protects streptozotocin-induced oxidative stress and beta-cell damage in rat pancreas. Pharmacol. Res., 2005, 51, 117-123.

7. Coxam V., Phyto-oestrogens and bone health. Proc. Nutr. Soc., 2008, 67, 184-95.

8. Fahmy S.R., Soliman A.M., Oxidative stress as a risk factor of osteoporotic by vitamin A in rats. Aust. J. Basic. Appl. Sci., 2009, 3, 1559-1568.

9. Henning P., Conaway H.H., Lerner U.H., Retinoid receptors in bone and their role in bone remodeling. Front Endocrinol (Lausanne)., 2015, 6:31. doi: 10.3389/fendo.2015.00031. eCollection 2015.

10. Hotchkiss C.E., Latendresse J., Ferguson S.A., Oral treatment with retinoic acid decreases bone mass in rats. Comp. Med., 2006, 56, 502-511.

11. Hozayen W.G., El-Desouky M.A., Soliman H.A., Ahmed R.R., Khaliefa A.K., Antiosteoporotic effect of Petroselinum crispum, Ocimum basilicum and Cichorium intybus L. in glucocorticoidinduced osteoporosis in rats. BMC Complement Altern. Med., 2016, Jun 2; 16, 165, doi: 10.1186/s12906-016-1140-y.

12. Huuskonen J., Arnala I., Olkkonen H., Alhava E., Alendronate influences bending force of femoral diaphysis after orchidectomy in rats. Ann. Chir. Gynaecol., 2001, 90, 109-114.

13. Inoue J., Choi J.M., Yoshidomi T., Yashiro T., Sato R., Quercetin enhances VDR activity, leading to stimulation of its target gene expression in Caco-2 cells. Nutr. Sci. Vitaminol. (Tokyo)., 2010, 56, 326-330.

14. Iwaniec U.T., Turner R.T., Smith B.J., Stoecker B.J., Rust A., Zhang B., Vasu V.T., Gohil K., Cross C.E., Traber M.G., Evaluation of long-term vitamin $\mathrm{E}$ insufficiency or excess on bone mass, density, and microarchitecture in rodents. Free. Radic. Biol. Med., 2013, 65, 1209-1214.

15. Johansson S., Melhus H., Vitamin A antagonizes calcium response to vitamin D in man. J. Bone. Miner. Res., 2001, 16, 1899-1905.

16. Johnell O., Kanis J.A., An estimate of the worldwide prevalence and disability associated with osteoporotic fractures. Osteoporos. Int., 2006, 17, 1726-1733.

17. Kneissel M., Studer A., Cortesi R., Susa M., Retinoid-induced bone thinning is caused by subperiosteal osteoclast activity in adult rodents. Bone, 2005, 36, 202-214.

18. Knežević A.H., Dikić D., Lisičić D., Kopjar N., Oršolić N., Karabeg S., Benković V., Synergistic effects of irinotecan and flavonoids on Ehrlich ascites tumour-bearing mice. Basic. Clin. Pharmacol. Toxicol., 2011, 109, 343-349.

19. Liang W., Luo Z., Ge S., Du J., Yang M., Yan M., Ye Z., Luo Z., Oral administration of quercetin inhibits bone loss in rat model of diabetic osteopenia. Eur. J. Pharmacol., 2011, 670, 317-324.

20. Liu R.H., Kang X., Xu L.P., Nian H.L., Yang X.W., Shi H.T., Wang X.J., Effects of the combined extracts of Herba epimedii and Fructus ligustri Lucidi on bone mineral content and bone turnover in osteoporotic rats. BMC Complement Altern. Med., 2015 Apr 9, 15, 112, doi: 10.1186/s12906-015-0641-4.

21. Mackinnon E.S., Rao A.V., Josse R.G., Rao L.G., Supplementation with the antioxidant lycopene significantly decreases oxidative stress parameters and the bone resorption marker N-telopeptide of type I collagen in postmenopausal women. Osteoporos. Int., 2011, 22, 1091-1101.

22. Manolagas S.C., Birth and death of bone cells: Basic regulatory mechanisms and implications for the pathogenesis and treatment of osteoporosis. Endocr. Rev., 2009, 21, 115-137.

23. Mukherjee M., Das A.S., Mitra S., Mitra C., Prevention of bone loss by oil extract of garlic (Allium sativum Linn.) in an ovariectomized rat model of osteoporosis. Phytother. Res., 2004, 18, 389-394.

24. Muthusami S., Ramachandran I., Muthusami B., Vasudevan G., Prabhu V., Subramaniam V., Jagadeesan A., Narasimha S., Ovariectomy induces oxidative stress and impairs bone antioxidant system in adult rats. Clinica. Chimica. Acta, 2005, 360, 81-86.

25. Nowak B., Matuszewska A., Filipiak J., Nikodem A., Merwid-Ląd A., Pieśniewska M., Kwiatkowska J., Grotthus B., Szeląg A.. The negative impact of selective activation of retinoic acid receptors on bone metabolism and bone mechanical properties in rats. Adv. Clin. Exp. Med., 2016, 25, 213-218.

26. Oršolić N., Benković V., Lisičić D., Dikić D., Erhardt J., Knežević A.H., Protective effects of propolis and related polyphenolic/flavonoid compounds against toxicity induced by irinotecan. Med. Oncol., 2010, 27, 1346-1358.

27. Oršolić N., Car N., Quercetin and hyperthermia modulate cisplatin-induced DNA damage in tumor and normal tissues in vivo. Tumour Biol., 2014, 35, 6445-6454.

28. Oršolić N., Gajski G., Garaj-Vrhovac V., Dikić D., Prskalo Z.Š., Sirovina D., DNA-protective effects of quercetin or naringenin in alloxan-induced diabetic mice. Eur. J. Pharmacol., 2011, 656, 110-118.

29. Oršolić N., Goluža E., Đikić D., Lisičić D., Sašilo K., Rođak E., Jeleč Z., Lazarus M.V., Orct T., Role of flavonoids on oxidative 
stress and mineral contents in the retinoic acid-induced bone loss model of rat. Eur. J. Nutr., 2014, 53, 1217-1227.

30. Oršolić N., Kunštić M., Kukolj M., Gračan R., Nemrava J., Oxidative stress, polarization of macrophages and tumour angiogenesis: Efficacy of caffeic acid. Chem. Biol. Interact., 2016, 256, 111-124.

31. Peng X., Jianfeng Y., Weizhang J., Qiankun C., Xio G., The effect of osteoporotic model rats induced by retinoic acid. Chinese Int. J. Traumatol., 2005, 4, 1-6.

32. Perazzella M.A., Markowitz G.S., Bisphosphonate nephrotoxicity. Kidney Int., 2008, 74, 1385-93.

33. Pitts C.J., Kearns A.E., Update on medications with adverse skeletal effects. Mayo Clin. Proc., 2011, 86, 338-343.

34. Prouillet C., Mazière J.C., Mazière C., Wattel A., Brazier M., Kamel S., Stimulatory effect of naturally occurring flavonols quercetin and kaempferol on alkaline phosphatase activity in MG-63 human osteoblasts through ERK and estrogen receptor pathway. Biochem. Pharmacol., 2004, 67, 1307-1313.

35. Rohde C.M., Manatt M., Clagett-Dame U., DeLuca H.F., Vita$\min \mathrm{A}$ antagonizes the action of vitamin D in rats. J. Nutr., 1999, 129, 2246-2250.

36. Sheweita S.A., Khoshhal K.I., Calcium metabolism and oxidative stress in bone fractures: role of antioxidants. Curr. Drug. Metab., 2007, 8, 519-525.

37. Togari A., Kondo M., Arai M., Matsumoto S., Effects of retinoic acid on bone formation and resorption in cultured mouse calvaria. Gen. Pharmacol., 1991, 22, 287-292.

38. Tsuji M., Yamamoto H., Sato T., Mizuha Y., Kawai Y., Taketani Y., Kato S., Terao J., Inakuma T., Takeda E., Dietary quercetin inhibits bone loss without effect on the uterus in ovariectomized mice. J. Bone. Miner. Metab., 2009, 27, 673-681.

39. Walker-Bone K., Recognizing and treating secondary osteoporosis. Nat. Rev. Rheumatol., 2012, 8, 480-492.

40. Wattel A., Kamel S., Prouillet C., Petit J.P., Lorget F., Offord E., Brazier M., Flavonoid quercetin decreases osteoclastic differentiation induced by RANKL via a mechanism involving NF kappa B and AP-1. J. Cell Biochem., 2004, 92, 285-295.

41. Wei M., Yang Z., Li P., Zhang Y., Sse W.C., Anti -osteoporosis activity of naringin in the retinoic acid-induced osteoporosis model. Am. J. Chin. Med., 2007, 35, 663-667.

42. Woo J.T., Nakagawa H., Notoya M., Yonezawa T., Udagawa N., Lee I.S., Ohnishi M., Hagiwara H., Nagai K., Quercetin suppresses bone resorption by inhibiting the differentiation and activation of osteoclasts. Biol. Pharm. Bull., 2004, 27, 504-509.

43. Xu Y.X., Wu C.L., Wu Y., Tong P.J., Jin H.T., Yu N.Z., Xiao L.W., Epimedium-derived flavonoids modulate the balance between osteogenic differentiation and adipogenic differentiation in bone marrow stromal cells of ovariectomized rats via Wnt $/ \beta$-catenin signal pathway activation. Chin. J. Integr. Med., 2012, 18, 909-917.

44. Yang J., Wu N., Peng J., Yang X., Guo J., Yin S., Wang J., Prevention of retinoic acid-induced osteoporosis in mice by isoflavoneenriched soy protein. J Sci Food Agric., 2016, 96, 331-338.

45. Zhao S., Niu .F, Xu C.Y., Liu Y., Ye L., Bi G.B., Chen L., Tian G., Nie T.H., Diosgenin prevents bone loss on retinoic acid-induced osteoporosis in rats. Irish J. Med. Sci., 2016, 185, 581-587.

Submitted: 11 January 2017. Revised: 21 May, 30 May, and 13 June 2017. Accepted: 4 July 2017. Published on-line: 6 November 2017. 